\title{
Phase calibration of spatial light modulators by means of
}

\section{Fresnel images}

Ll Martínez-León ${ }^{1}$, Z Jaroszewicz ${ }^{2}$, A Kołodziejczyk ${ }^{3}$, V Durán $^{1}$, E Tajahuerce ${ }^{1}$, and $\mathbf{J}$ Lancis ${ }^{1}$

${ }^{1}$ GROC·UJI, Departament de Física, Universitat Jaume I, 12071 Castelló, Spain, and

INIT (Institute of New Imaging Technologies), Universitat Jaume I, 12071 Castelló, Spain

${ }^{2}$ Institute of Applied Optics, Kamionkowska 18, 03-805 Warsaw, Poland, and

National Institute of Telecommunications, Szachowa 1, 04-894 Warsaw, Poland

${ }^{3}$ Faculty of Physics, Warsaw University of Technology, Koszykowa 75, 00-662 Warsaw, Poland

E-mail: mmtzjaroszewicz@post.pl

\begin{abstract}
Reliable application of spatial light modulators (SLMs) as programmable diffractive optical elements requires a thorough calibration. In this paper, we propose a method for calibrating SLMs based on the evaluation of Fresnel images. Fresnel images generated by a binary phase diffraction grating consist of binary irradiance distributions whose visibility depends on the phase modulation. By displaying on our device a diffraction grating with a binary phase step along one direction and a linearly increasing phase along the orthogonal direction, we perform a complete phase calibration. In this way, the phase modulation for every pixel and for every value of the entrance signal can be determined experimentally. Additionally, data acquisition can be significantly simplified with this scheme.
\end{abstract}

Keywords: diffraction gratings, Talbot effect, liquid crystal devices, spatial light modulators

PACS: 42.79.Kr Display devices, liquid-crystal devices, 42.79.Dj Gratings,

42.25.Fx Diffraction and scattering 


\section{Introduction}

In the last few decades, spatial light modulators (SLMs) have become fundamental elements in multiple optical applications [1]. At present, the most common SLMs are those based in liquid crystal (LC) technology, which consist of an array of cells (pixels) with a LC film whose molecular alignment can be modified by a driven voltage. LC-SLMs are polarization-sensitive devices, i.e., they primarily modulate the state of polarization (SOP) of an input beam. This change in the SOP can be converted into a complex amplitude modulation by sandwiching the LC-SLM between polarization elements, as linear polarizers and retarder plates $[2,3]$.

In numerous applications, the design of programmable diffractive optical elements (DOEs) for a spatial and temporal control of the phase of an input light wavefront is required. Some LC-SLMs are specifically designed for this task, as those composed by parallel aligned (PAL) nematic LC cells [4]. In contrast, off-the-shelf twisted nematic LC displays (TNLCDs) provide a coupled intensity and phase modulation, so they must previously undergo an optimization process [5-7]. In all cases, it is critical to perform an experimental determination of the spatial phase distribution of the SLM as a function of the parameter that controls the signal applied to the device (typically, the gray-level of the image displayed by commercial LC devices). A lack of spatial uniformity in the phase response of a SLM can appear due to addressing errors or to factors that are voltage-independent, as, for example, the curvature of the surface device, which causes variations in the LC thickness [8-10]. In the case of widespread used liquid crystal on silicon (LCoS) displays, the reflected wavefronts can be distorted by the curvature of the silicon backplane [9-11]. Typical magnitudes of this kind of phase errors are of the order of $\lambda$ over the aperture of the device.

Many phase calibration methods of SLMs have been reported in the past. It is apparent that the phase modulation curve of a SLM can be directly measured with the aid of an interferometer. Moreover, it is also possible to study spatial phase distortions along the device aperture with interferometric methods, as those based on the analysis of Fizeau-type fringes [12], or on the use of a Twyman-Green 
interferometer [11] or a Mach-Zehnder interferometer [13] with a four-phase step shift equipment added [10]. The main drawback of this kind of systems is its high sensitivity to mechanical vibrations, as well as the large number of optical components that are required.

An alternative to calibration techniques based on the interference by amplitude division is the use of the double-slit interference principle. Thereby, the phase response of a TNLCD has been determined by the measurement of the lateral displacement of the interference pattern produced in a Young type experiment. For this purpose, a mask with two pinholes is placed behind the display. Each pinhole covers a portion of the SLM with a different value of the cell steering parameter [14-16].

Another group of calibration techniques retrieve the phase information from the analysis of farfield diffraction intensity distributions when certain selected patterns are implemented onto the SLM. Thus, the phase response of a liquid crystal display has been characterized by the measurement of the diffraction efficiency when series of binary phase gratings with different, albeit constant, phase step heights are programmed on the device [17]. In this way it is also possible to detect a period asymmetry of the programmed gratings by measuring differences between diffraction efficiencies of two conjugate orders [18]. However, the accuracy of such techniques, as based on intensity measurements, is rather moderate and secondly, is affected by the existence of residual intensity modulation or by the contribution to the zero-order intensity due to the pixelated structure of the device. These artifacts have been recently faced by Engstrom et al. [19].

Here, we focus our attention on a couple of techniques based on the fractional Talbot effect [20]. It was shown by Guigay [21] and later found independently [22-24], that the Fresnel images of binary phase gratings, at multiples of certain fractions of the Talbot distance, exhibit binary intensity distributions, whose visibility depends on the phase step height. This property of Fresnel images has found numerous applications in various areas of physics [24-27]. In the context of SLM calibration, the phase response of a TNLCD has been measured by displaying binary phase gratings of constant phase step height [23]. 
In the present paper, we propose a modification of the last approach, which relies on the implementation of a phase grating with a binary phase step along one direction and a linearly increasing phase along the orthogonal direction. Thanks to this change, it is possible to observe the resulting distribution of the visibility function with a kind of carrier frequency fringes, and not in the uniform field, as it has took place in some of the previous approaches. It should be noted that the other aforementioned non interferometer-based techniques are of integral character, i.e., they allow determining the calibration function in average for the whole device rather than for every pixel separately. However, the present approach enables, as we will demonstrate, to detect and measure the local addressing errors. In this way, it is possible to check the degree of spatial uniformity of the SLM phase response with a very simple experimental set-up.

\section{Fresnel images of binary phase gratings}

The method proposed is based on the projection of a phase grating on a SLM and the analysis of the resultant Fresnel images produced by the modulated light beam. Let us assume that a SLM, where a binary phase grating with period $d$ has been implemented, is illuminated by a divergent spherical wave with wavelength $\lambda$ and its focus $S$ placed at a distance $z_{0}$ from the display, as shown schematically in Fig. 1.

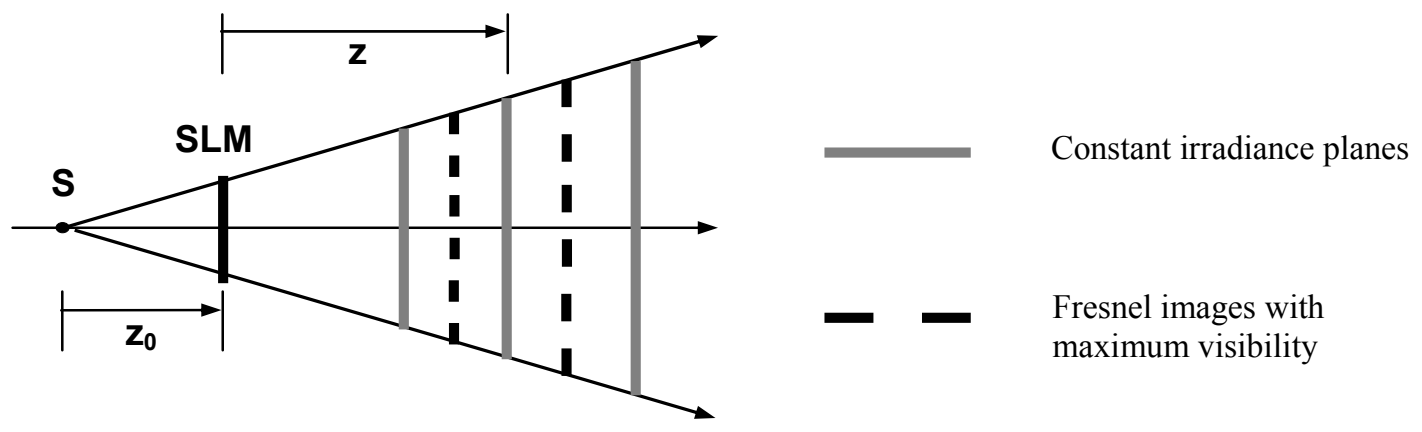

Figure 1: Fresnel images of the SLM binary phase grating illuminated by a spherical wave with radius of curvature equal to $z_{0}$ in the plane of the grating. 
According to the results obtained for example in ref. [24], it can be seen that for distances

$$
\mathrm{z}=\frac{\mathrm{f} \mathrm{z}_{0}}{\mathrm{z}_{0}-\mathrm{f}}
$$

replicas of the entrance grating magnified by a factor $M=\left(z+z_{0}\right) / z_{0}$ will appear. Here, $f=m D$, where $m$ is an integer and $D$ stands for the selfimage distance equal to $2 d^{2} / \lambda$ [28]. Analogously, at planes determined by Eq. (1), with $f=(m+1 / 2) D$, self-images displaced by half a period will appear. Irradiance in both sets of planes is obviously constant. In turn, at distances $z$ such that $f=(m+1 / 4) D$ or $f=(m+$ 3/4) $D$ binary Fresnel images can be observed, with the visibility function determined by [24]

$$
V=\frac{I_{2}-I_{1}}{I_{2}+I_{1}}=\sin \varphi
$$

where $\varphi$ is the phase step height and $I_{1}, I_{2}$ are intensities of even and odd semiperiods, respectively. The negative values of $\sin \varphi$ should be interpreted as a contrast reversal of the intensity pattern in the Fresnel image planes.

In general, Fresnel images appear at planes $z$ corresponding to $f=(m+p / q) D$ where $p$ and $q$ are integers with no common factor and $p<q$, but $p / q=1 / 4$ or $3 / 4$ provides the best visibility for our application. Visibility is reduced for $p / q$ other than $1 / 4$ or $3 / 4$ [28], e.g., for $1 / 8,3 / 8,5 / 8$ and $7 / 8$ by a factor of $2^{1 / 2}$ [24]. As can be seen, use of a spherical illuminating wave allows for a flexible choice of the magnification and position of the Fresnel images.

\section{Fresnel images of gratings with variable phase step}

In our scheme, a phase grating with a binary phase step along one direction $(O X)$, and a linearly increasing phase along the orthogonal direction $(O Y)$ is programmed, according to the following formula for one unit cell (see Fig. 2):

$$
t_{P}(x, y)=\operatorname{rect}\left[\frac{(x-d / 4)}{d / 2}\right]+\operatorname{rect}\left[\frac{(x-3 d / 4)}{d / 2}\right] \exp \left(i \varphi_{\max } y / D_{y}\right),
$$


where $\varphi_{\max }$ is the maximum phase change obtainable by the SLM device and $D_{y}$ is the length of the grating in the $O Y$ direction on the display.

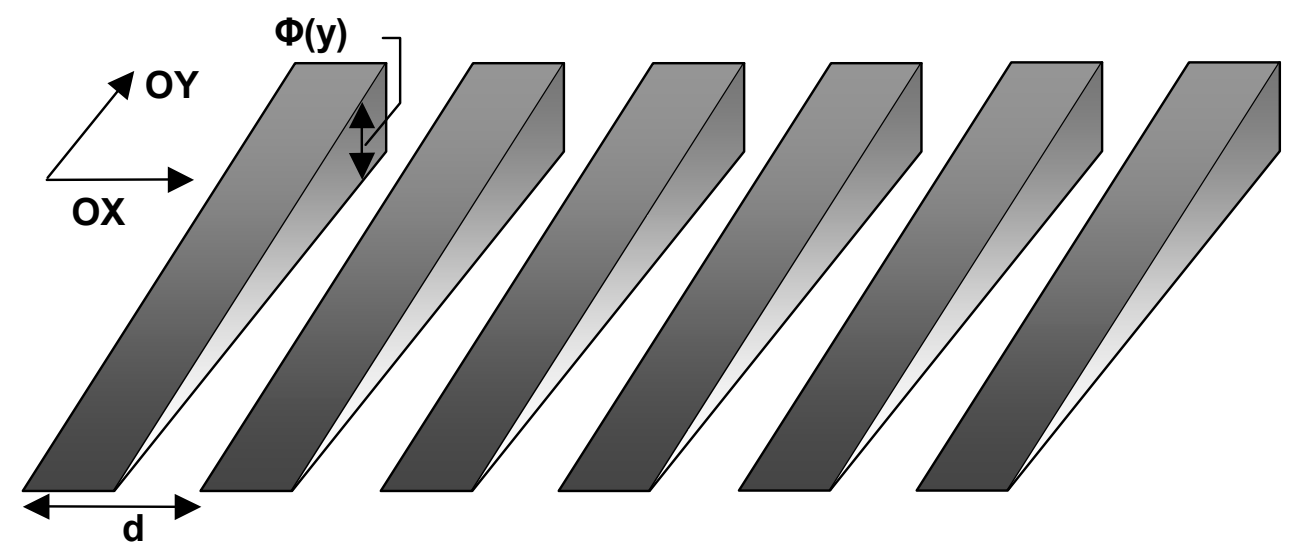

Figure 2: Binary phase grating with the height of the phase steps determined by Eq. (3).

If the grating were perfect, then the visibility function of the Fresnel image should obey the following relationship

$$
V=\frac{I_{2}-I_{1}}{I_{2}+I_{1}}=\sin \left(\varphi_{\max } y / D_{y}\right)
$$

The resulting visibility function pattern would be a set of straight lines resembling interference fringes created by two plane waves inclined under an angle $\varphi_{\max } / k D_{y}$. A simulation of the intensity distribution at the output plane of the ideal case for a grating with $D_{y}=20 \mathrm{~mm}, d=0.8 \mathrm{~mm}$ and $\varphi_{\max }=2 \pi$, modulating a parallel light beam is shown in Fig. 3.

From theoretical descriptions of the Fresnel images $[19,29,30]$ it results that the complex amplitude distribution in the plane of the Fresnel image can be considered as a sum of mutually displaced replicas of the entrance grating with additional specific phase factors. In particular, a transparent semiperiod of one replica overlaps in the Fresnel image plane with the semiperiod containing the phase step of the second replica and conversely. Therefore, only one image will be enough in order to obtain the whole map of the given entrance signal non-accuracies. Evidently, in order to cover all pixels of the SLM 
separately and to obtain the complete calibration set, it is necessary to implement a grating with the minimum period, i.e., twice as wide as the distance between the pixels.

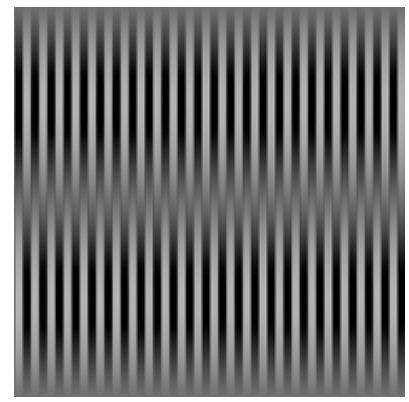

(a)

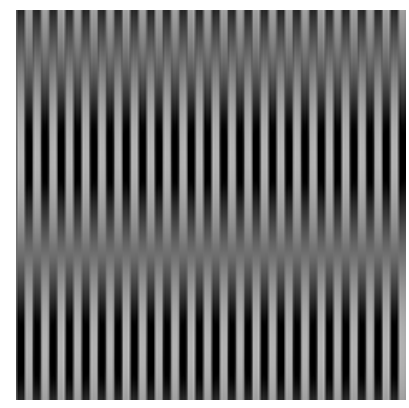

(b)

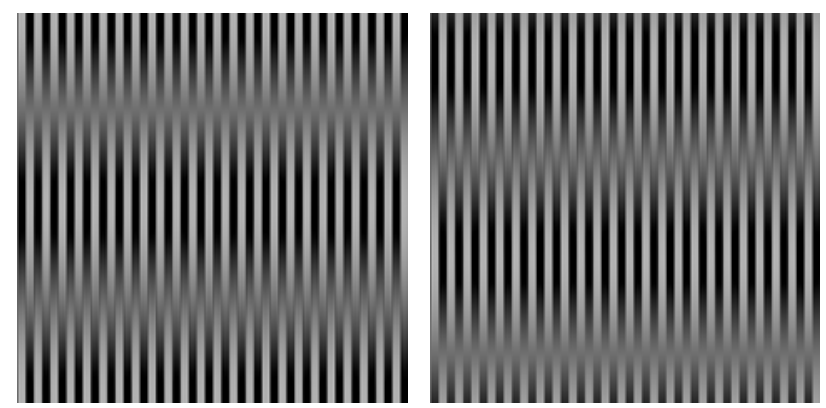

(c)

(d)

Figure 3: Simulated intensity distributions in the Fresnel plane of the modified binary phase grating with phase steps determined according to Eq. (5) for $N=48$ : (a) $n=0,24$; (b) $n=6 ; 30$ (c) $n=12,36$; (d) $n=$ 18,42 .

A further step in calibration involves checking the SLM response when different entrance signals are applied to the same pixel. This is easily achieved if the grating becomes variable by displaying a sequence of $N$ gratings displaced along the $O Y$ axis by half a period, according to the following formula:

$$
t_{P, n}(x, y)=\operatorname{rect}\left[\frac{(x-d / 4)}{d / 2}\right]+\operatorname{rect}\left[\frac{(x-3 d / 4)}{d / 2}\right] \exp \left(i \varphi_{\max }(y-n d / 2) / D_{y}\right) \text {, }
$$

with $n$ integer equal to $0,1, \ldots, \mathrm{N}-1$ and $N=2 D_{y} / d$. Figures from $3 \mathrm{a}$ to $3 \mathrm{~d}$ show a set of 4 simulated intensity distributions in the Fresnel plane, when displaying a sequence of modified binary phase gratings displaced by $D_{y} / 8$. 


\section{Experimental results}

We have checked experimentally the phase modulation attained by a PAL-SLM, Hamamatsu X8267, when displaying variable phase step binary gratings. This device is designed to achieve a high-efficiency phase-only modulation. The PAL-SLM is addressed optically through a supplementary electrically addressable LCD but, thanks to the coupling-optics design, the pixel structure of the LCD is not transferred into the PAL-SLM.

This display consists of $768 \times 768$ pixels, with a maximum resolution of $19 \mathrm{lp} / \mathrm{mm}$. In our experiments, a single semiperiod was 16 pixels wide. We illuminated the SLM with a parallel light beam, with $\lambda=514.5 \mathrm{~nm}$.

The set of intensity distributions presented in Fig. 4 shows the experimental Fresnel images corresponding to the simulated patterns in Fig. 3. These images are a sample within the possible range of phase measurements, chosen to show the effect of a $D_{y} / 4$ grating displacement. The straight dark segment in Figs $3 b, 3 c$, and $3 d$ corresponds to the phase discontinuity $0-2 \pi$ displacing according to Eq. (5). As can be seen in Fig. 4, the modulation exceeds $2 \pi$. Besides, curved fringes are produced, instead of horizontal straight ones. It clearly indicates a different phase change produced by the same value of the entrance signal in different positions of the device. The displaced gratings displayed on the SLM permit a detailed analysis of the complete area of the modulator.

Actually, our experimental Fresnel images have provided the necessary information to describe the phase modulation in any region of the device. In particular, we have determined the visibility function in almost 200 positions over the display, and have obtained the corresponding calibrations curves. The calibration procedure begins with the processing of intensity distributions like those shown in Fig. 4. The visibility is computed for a particular position. The possibility of considering a region of several pixels in the camera minimizes the experimental error. Then, phase values are extracted through Eq. (4). Each set of data is fitted to take into account overall intensity values. It is worth remembering that the method relies on local visibility variations, which give information about the phase response for each region of the SLM. 
As an example, in Fig. 5 we present the phase modulation at equispaced points along two different lines in the OY direction. These data have been extracted from the Fresnel image resulting from a single grating, such as any of the individual images in Fig. 4.

Also, we have computed the phase modulation at the chosen positions when varying the entrance signal. This signal was changed by displaying on the SLM different displaced gratings with an increasing phase along the OY direction. As a sample, Fig. 6 shows the phase modulation at two different positions on the modulator. It is a function of the gray level displayed in each image sent to the SLM at those particular positions. Experimentally, we have processed the series of images recorded when displacing the grating by $D_{y} / 16$. Both figures represent exemplary cross-sections of the complete calibration data, which should contain phase modulations that can be obtained for every pixel and all values of the entrance signals. Local addressing errors can be not only detected but also measured thanks to the sort of information supplied by the two previous figures. The typical phase determination error was within the range of $\pm 0.3 \mathrm{rad}$.

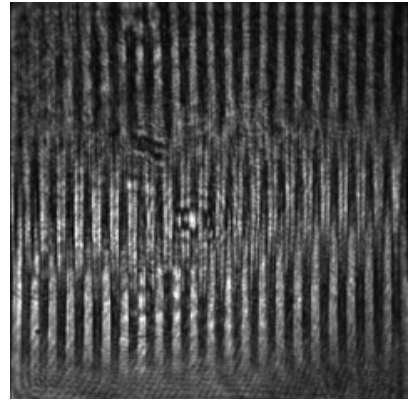

(a)

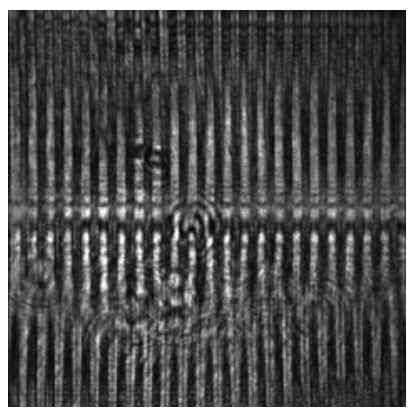

(c)

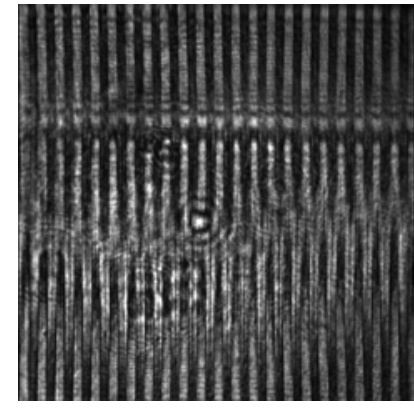

(b)

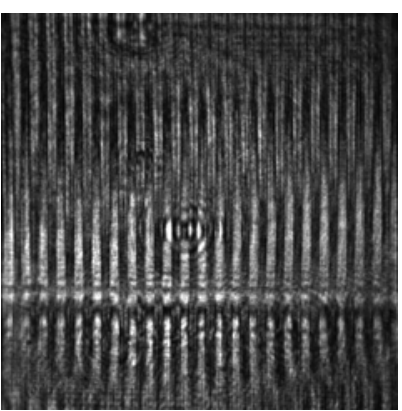

(d) 
Figure 4: Experimental results showing the Fresnel images of the modified binary phase grating for $\mathrm{N}=$ 48: (a) $n=0 ;$ (b) $n=12 ;$ (c) $n=24 ;$ (d) $n=36$.

It is worth to remark that inspection of Fresnel images allows the observation and measurement of calibration errors only. For the substrate errors of the SLM or aberrations of the remaining elements of the set-up, the system acts as a Talbot interferometer [19], [31]. Therefore, any errors of this kind remain only in a residual quantity. Strictly speaking, a lateral derivative of this error is added to the observed pattern. Taking into account that the typical errors due to the curvature are of the order of $\lambda$ over the area of a typical SLM [9-12], and also that a typical aperture can contain approximately 1000 elementary cells in one direction, the magnitude of this error over one elementary cell can be evaluated as being of order of $\lambda / 1000$.

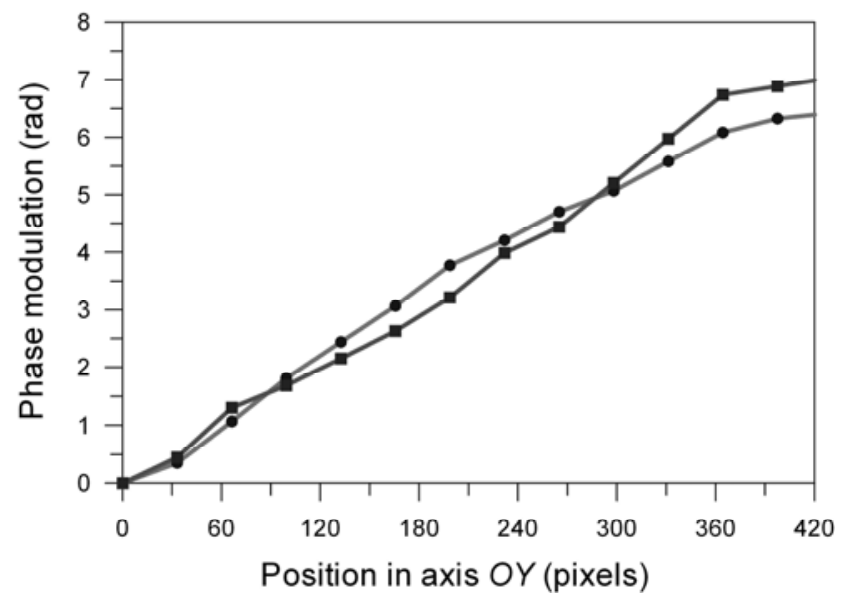

Figure 5: Phase modulation along two different lines in the OY direction. 


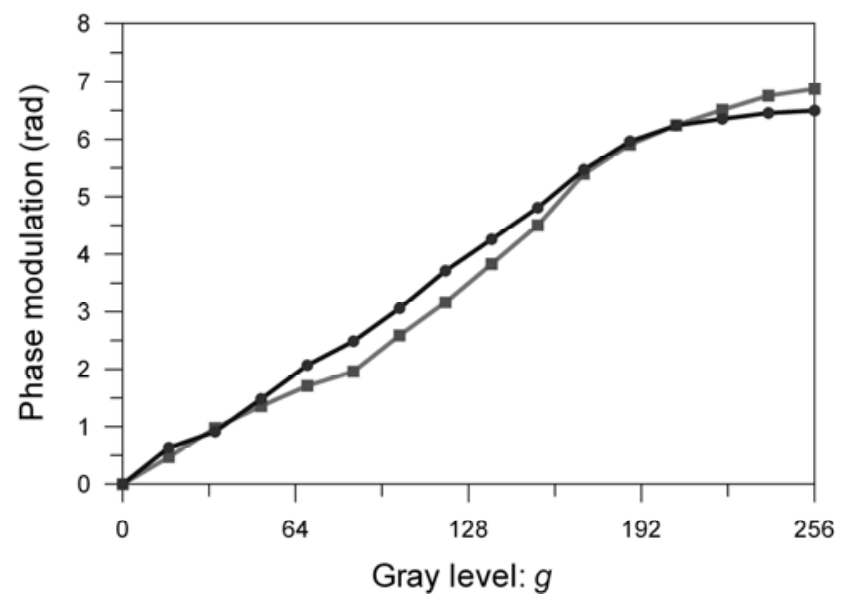

Figure 6: Phase modulation at two different positions for varying entrance signals.

As an important practical consideration, the observation should be performed in the first Fresnel plane, where the shear value is the smallest and thereby this residual phase error is also minimum.

\section{Conclusions}

A method for SLM phase calibration, by inspection of Fresnel images produced by gratings, for all pixels and all steering signal values, has been shown and demonstrated experimentally. The scheme is quasiindependent of the phase errors introduced by the varying thickness of the liquid crystal layer and represents a suitable method for the evaluation of the addressing errors. A complete SLM calibration can be performed if the period of the grating is chosen equal to two pixels. Then, in principle, a single Fresnel image of a phase grating with linear increase of the phase step along its length should be sufficient for recovering the calibration data for all device pixels and all input data range. By moving the phase profile along the grating period it is possible to gather all phase values for all input signals and for every pixel in a simplified manner.

\section{Acknowledgments}


Authors acknowledge financial support from Fundació Caixa Castelló-Bancaixa (project P1·1B2006-29) and from the Spanish Ministerio de Educación y Ciencia (Consolider Programme SAUUL CSD200700013). This work was also supported by the Polish Ministerstwo Nauki i Informatyzacji under contracts 4 T07D 00329 and R04 02503. 


\section{References}

[1] Efron U ed. 1994 Spatial Light Modulator Technology (New York: Dekker)

[2] de Bougrenet de la Tocnaye J L and Dupont L 1997 Complex amplitude modulation by use of liquid-crystal spatial light modulators Appl. Opt. 36 1730-1741

[3] Duran V, Lancis J, Tajahuerce E and Jaroszewicz Z 2006 Equivalent retarder-rotator approach to on-state twisted-nematic liquid crystal displays J. Appl. Phys. 99113101

[4] Mukohzaka N, Yoshida N, Toyoda H, Kobayashi Y and Hara T 1994 Diffraction Efficiency Analysis of a Parallel-Aligned Nematic-Liquid-Crystal Spatial Light-Modulator Appl. Opt. 33 2804-2811

[5] Konforti N, Marom E and Wu S T 1998 Phase-Only Modulation with Twisted Nematic LiquidCrystal Spatial Light Modulators Opt. Lett. 13 251-253

[6] Duran V, Lancis J, Tajahuerce E and Fernández-Alonso M 2006 Phase-only modulation with a twisted nematic liquid crystal display by means of equi-azimuth polarization states Opt. Express 14 56075616

[7] Clemente P, Durán V, Martínez-León L, Climent V, Tajahuerce E and Lancis J 2008 Use of polar decomposition of Mueller matrices for optimizing the phase response of a liquid-crystal-on-silicon display Opt. Express 16 1965-1974

[8] Cho D J, Thurman S T, Donner J T and Morris G M 1998 Characteristics of a $128 \times 128$ liquidcrystal spatial light modulator for wave-front generation Opt. Lett. 23 969-971

[9] Otón J, Ambs P, Millán M S and Pérez-Cabré E 2007 Multipoint phase calibration for improved compensation of inherent wavefront distortion in parallel aligned liquid crystal on silicon displays Appl. Opt. $465667-5679$

[10] Xun X and Cohn R W 2004 Phase calibration of spatially nonuniform spatial light modulators Appl. Opt. 43 6400-6406 
[11] Zhang $\mathrm{H}$, Zhang $\mathrm{J}$ and $\mathrm{Wu} \mathrm{L}, 2007$ Evaluation of phase-only liquid crystal spatial light modulator for phase modulation performance using a Twyman-Green interferometer 2007 Meas. Sci. Technol. 18 $1724-1728$

[12] Grother P and Casasent D 2001 Optical path difference measurement techniques for SLMs 2001 Opt. Commun. 189 31-38

[13] Mok F, Diep J H, Liu H-K and Psaltis D 1986 Real-time computer-generated hologram by means of liquid-crystal television spatial light modulator Opt. Lett. 11 748-750

[14] Bergeron A J, Gauvin F, Gagnon D, Gingras H, Arsenault H H and Doucet M 1995 Phase calibration and applications of a liquid-crystal spatial light modulator Appl. Opt. 34 5133-5139

[15] Florence J.M. and Juday R.D. 1991 Full-complex modulation with two one-parameter SLMs Wave Propagation and Scattering in Varied Media II (San Diego, CA, USA, July 1991); Proc. SPIE 1558, 499504.

[16] Dou R and Giles M K 1996 Simple technique for measuring the phase property of a twisted nematic liquid crystal television Opt. Eng. 35 808-812

[17] Zhang Z, Lu G and Yu F 1994 Simple method for measuring phase modulation in liquid crystal television Opt. Eng. 33 3018-3022

[18] Hermerschmidt A, Osten S, Krüger S, and Blümel T 2007 Wave front generation using a phaseonly modulating liquid crystal-based micro-display with HDTW resolution Adaptive Optics for Laser Systems and Other Applications (Prague, Czech Republic, April 2007); Proc. SPIE 6584 65840E

[19] Engström D, Milewski G, Bengtsson J and S. Galt S 2006 Diffraction-based determination of the phase modulation for general spatial light modulators Appl. Opt. 45 7195-7204

[20] Patorski K 1989 The self-imaging phenomenon and its applications Progr Opt. 27 1-108

[21] Guigay J P 1971 On Fresnel diffraction by one-dimensional periodic objects, with application to structure determination of phase objects Opt. Acta 18 677-682

[22] Arrizon V and Ojeda-Castañeda J 1992 Irradiance at Fresnel planes of a phase grating J. Opt. Soc. Am. A 9 1801-1806 
[23] Serrano-Heredia G, Lu G, Purwosumarto P and Yu F T S 1996 Measurement of the phase modulation in liquid crystal television based on the fractional-Talbot effect Opt. Eng. 35 2680-2684

[24] Jaroszewicz Z, Kołodziejczyk A, Kowalik A, and Restrepo R 2000 Determination of the step height of the binary phase grating from its Fresnel images Optik 111 207-210

[25] Spence J C H and Qian W 1992 Irradiance at Fresnel planes of a phase grating Phys. Rev. B 45 $10271-10280$

[26] Cloetens P, Guigay J P, De Martino C, Baruchel J and Schlenker M 1997 Fractional Talbot imaging of phase gratings with hard x rays Opt. Lett. 22 1059-1061

[27] Riley Jr. W A 1980 Optical determination of low ultrasonic powers J. Acoust. Soc. Am. 671386 1388

[28] Cowley J M and Moodie A F 1960 Fourier Images IV: The Phase Grating Proc. Phys. Soc. 76 378384

[29] Kolodziejczyk A 1988 Self-imaging effect - a new approach Opt. Commun. 65 84-86

[30] Banaszek K, Wodkiewicz K and Schleich W P 1998 Fractional Talbot effect in phase space: A compact summation formula Opt. Express 2 169-172

[31] Silva D E 1972 Talbot interferometer for radial and lateral derivatives Appl. Opt. 11 2613-2624 